\title{
Parallel Space-Time Kernel Density Estimation
}

\author{
Erik Saule ${ }^{\dagger}$, Dinesh Panchananam ${ }^{\dagger}$, Alexander Hohl ${ }^{\ddagger}$, Wenwu Tang ${ }^{\ddagger}$, Eric Delmelle \\ ${ }^{\dagger}$ Dept. of Computer Science, ${ }^{\ddagger}$ Dept. of Geography and Earth Sciences \\ UNC Charlotte \\ Email: \{esaule,dpanchan,ahohl,wtang4,eric.delmelle\}@uncc.edu
}

August 24, 2021

\begin{abstract}
The exponential growth of available data has increased the need for interactive exploratory analysis. Dataset can no longer be understood through manual crawling and simple statistics. In Geographical Information Systems (GIS), the dataset is often composed of events localized in space and time; and visualizing such a dataset involves building a map of where the events occurred.

We focus in this paper on events that are localized among three dimensions (latitude, longitude, and time), and on computing the first step of the visualization pipeline, space-time kernel density estimation (STKDE), which is most computationally expensive. Starting from a gold standard implementation, we show how algorithm design and engineering, parallel decomposition, and scheduling can be applied to bring near real-time computing to space-time kernel density estimation. We validate our techniques on real world datasets extracted from infectious disease, social media, and ornithology.
\end{abstract}

\section{Introduction}

The rapid propagation of infectious diseases (e.g. zika, Ebola, H1N1, dengue fever) is conducive to serious, epidemic outbreaks, posing a threat to vulnerable populations. Such diseases have complex transmission cycles, and effective public health responses require the ability to monitor outbreaks in a timely manner [EE11]. Space-time statistics facilitate the discovery of disease dynamics including rate of spread, seasonal cyclic patterns, direction, intensity (i.e. clusters), and risk of diffusion to new regions. However, obtaining accurate results from space-time statistics is computationally very demanding, which is problematic when public health interventions are promptly needed. The issues of computational efforts are exacerbated with spatiotemporal datasets of increasing size, diversity and availability [GWM14]. High-performance computing reduces the effort required to identify these patterns, however heterogeneity in the data must be accounted for [HDTC16].

Epidemiology is only one of the application domains where it is important to understand how events occurring at different locations and different times form clusters. Political analysis, social media analysis, or the study of animal migration also require the understanding of spatial and temporal locality of events. The massive amount of data we see nowadays is often analyzed using complex models. But before these can be constructed, data scientists need to interactively visualize and explore the data to understand its structure.

In this paper, we present the space-time kernel density estimation application which essentially builds a 3D density map of events located in space and time. This problem is computationally expensive using existing algorithms. That is why we developed better sequential algorithms for this problem that reduced the complexity by orders of magnitude. And to bring the runtime in the realm of near real-time, we designed parallel strategies for shared memory machines.

Section 2 presents the STKDE application along with a reference implementation that uses a voxel-based algorithm VB to compute STKDE. In Section 3, we investigate the sequential problem and develop a point-based algorithm PB that significantly reduce the complexity compared to VB. We also identify invariants linked to the structure of the kernel density estimate function that allows to reduce the computational cost by an additional factor and build the 
PB-SYM algorithm based on that observation. We develop two parallelization strategies (PB-SYM-DR and PB-SYMDD) in Section 4 that are designed to make the computation pleasingly parallel. However both strategies are not work-efficient and can cause significant work overhead in some cases. That is why we introduce PB-SYM-PD in Section 5 that provide a work-efficient decomposition of the points. However that algorithm has internal dependencies which can induce a long critical path and cause load imbalance; and we develop PB-SYM-PD-SCHED to leverage alternative ordering of the work to reduce the critical path. To reduce the critical path further, we propose the PBSYM-PD-REP algorithm which introduces some work overhead only where is needed to achieve a short critical path. Section 6 presents experimental results on 4 different datasets and 21 instances extracted from these datasets. The experiments highlights that the sequential strategies are efficient. The parallel strategies have their pros and cons and different strategies appear to be best in different scenarios. Section 7 discusses the applicability of related problems and techniques.

\section{Space-Time Kernel Density Estimation}

\subsection{Description}

Space-time kernel density (STKDE) is used for identifying spatiotemporal patterns in datasets. It is a temporal extension of the traditional 2D kernel density estimation [Sil86] which generates density surface ("heatmap") from a set of $n$ points located in a geographic space. The resulting density estimates are visualized within the space-time cube framework using two spatial $(x, y)$ and a temporal dimension $(t)$ [NY10]. STKDE creates a discretized 3D volume where each voxel (3D equivalent of a pixel) is assigned a density estimate based on the surrounding points. The space-time density is estimated using (following the notations of [HDTC16]):

$$
\hat{f}(x, y, t)=\frac{1}{n h_{s}^{2} h_{t}} \sum_{i \mid d_{i}<h_{s}, t_{i}<h_{t}} k_{s}\left(\frac{x-x_{i}}{h_{s}}, \frac{y-y_{i}}{h_{s}}\right) k_{t}\left(\frac{t-t_{i}}{h_{t}}\right)
$$

Density $\hat{f}(x, y, t)$ of each voxel is determined by number and distance of events (points) $\left(x_{i}, y_{i}, t_{i}\right)$ within its vicinity, which is conceptualized by a cylinder. The spatial bandwidth $h_{s}$ forms a circle which, due to the orthogonal relationship between space and time, is extended to a cylinder by temporal bandwidth $h_{t}$. For every event inside the cylinder, the spatial $\left(d_{i}\right)$ and temporal $\left(t_{i}\right)$ distances are smaller than $h_{s}$ and $h_{t}$. Therefore, the event receives a weight based on the kernel functions $k_{s}$ and $k_{t}$ (distance decay):

$$
\begin{gathered}
k_{s}(u, v)=\frac{\pi}{2}(1-u)^{2}(1-v)^{2} \\
k_{t}(w)=\frac{3}{4}(1-w)^{2}
\end{gathered}
$$

Figure 1 illustrates how varying the bandwidths used in STKDE helps focusing the graphical visualization of Dengue fever cases in Cali, Colombia [DDC $\left.{ }^{+} 14\right]$. Figure 2 shows the impact of a single point on the neighboring space.

Computationally, the domain of size $g_{x}, g_{y}, g_{t}$ is discretized in voxels using a spatial resolution sres and a temporal resolution tres. Therefore, the domain is represented by a grid of size $G_{x}=\left\lceil\frac{g_{x}}{\text { sres }}\right\rceil, G_{y}=\left\lceil\frac{g_{y}}{\text { sres }}\right\rceil, G_{t}=\left\lceil\frac{g_{t}}{\text { tres }}\right\rceil$. Each point causes a density increase in the voxels that are within a cylinder centered on the point, of radius equal to the spatial bandwidth in voxels $H_{s}=\left\lceil\frac{h_{s}}{s r e s}\right\rceil$, and of half height equal to the temporal bandwidth $H_{t}=\left\lceil\frac{h_{t}}{\text { tres }}\right\rceil$.

All the notations are summarized in Table 1. As a convention all uppercase notations denote quantities in voxels and all lowercase notations denote quantities in domain space.

\subsection{Gold Standard Implementation}

The gold standard implementation (for instance from [HDTC16]) follows the exact definition of STKDE as it is given above. It is a voxel-based algorithm we call VB. For each voxel, VB finds the points within the temporal and spatial bandwidths and calculates the contribution to the density of this voxel. The pseudo code is given in Algorithm 1. 


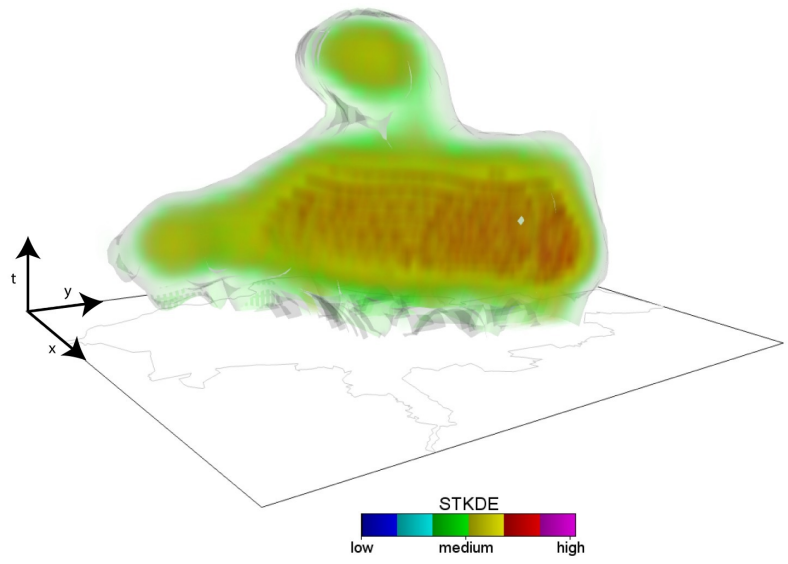

(a) $h_{s}=2500 m, h_{t}=14$ days

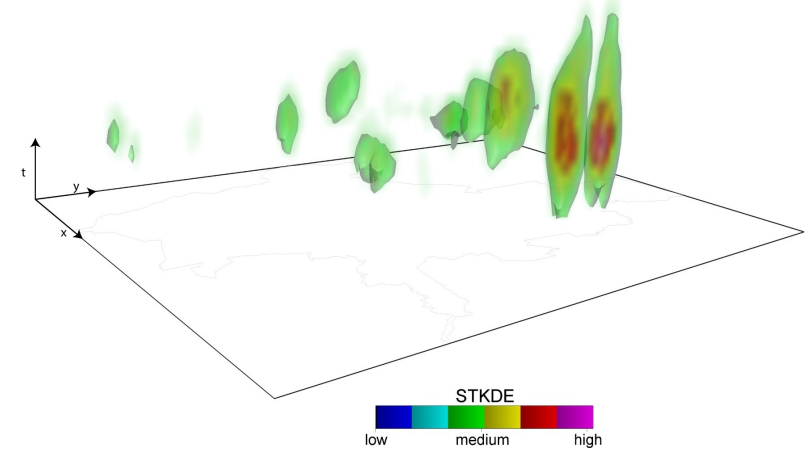

(b) $h_{s}=500 \mathrm{~m}, h_{t}=7$ days

Figure 1: Visualization of Dengue fever cases in Cali, Colombia in 2010 and 2011 for different spatial bandwidth and temporal bandwidth.

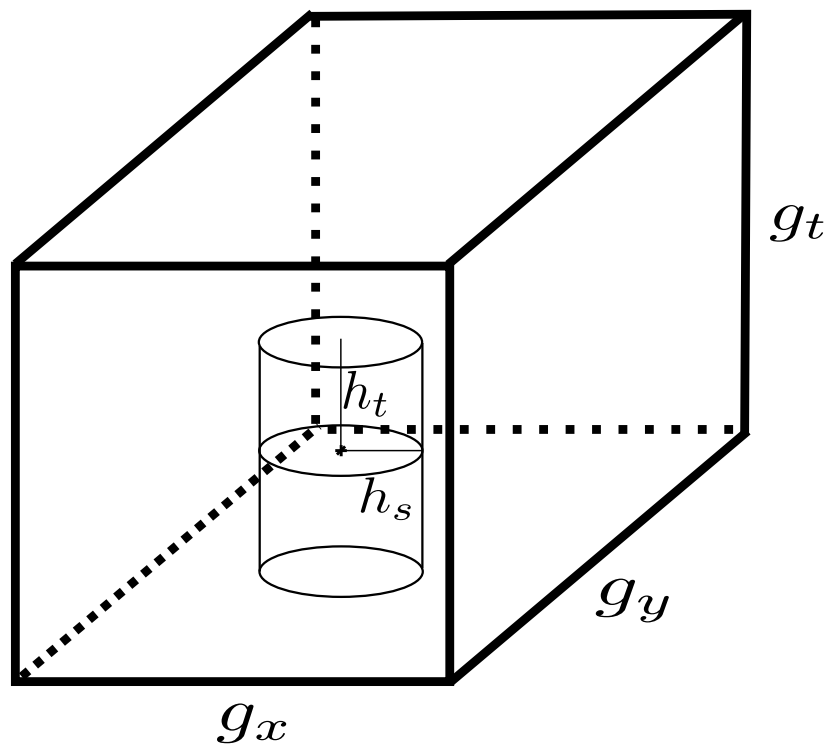

Figure 2: The computation of STKDE happens in a domain space of size $g_{x}, g_{y}, g_{t}$. Each point impacts the neighboring space at a distance $h_{s}$ in space and $h_{t}$ in time; forming a cylinder of diameter $2 h_{s}$ and of height $2 h_{t}$.

\begin{tabular}{|l|l|}
\hline$n$ & Number of points \\
$s=(x, y, t)$ & A voxel and sampling coordinate \\
$\left(x_{i}, y_{i}, t_{i}\right)$ & Coordinate of point $i$ \\
$h_{s}, h_{t}$ & Spatial and temporal bandwidth \\
$g_{x}, g_{y}, g_{t}$ & Real size of the domain (in meters) \\
\hline sres, tres & Resolution (in meters) \\
$s=(X, Y, T)$ & A voxel in voxel space \\
$\left(X_{i}, Y_{i}, T_{i}\right)$ & Voxel of point $i$ \\
$G_{x}, G_{y}, G_{t}$ & Size of the domain (in voxels) \\
$H_{s}, H_{t}$ & Bandwidth (in voxels) \\
\hline
\end{tabular}

Table 1: Notations 


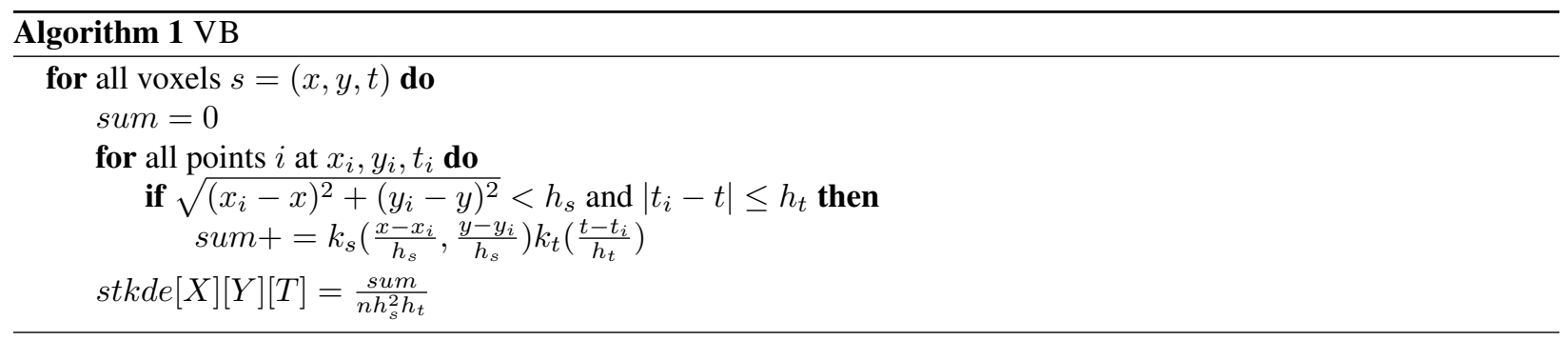

The algorithm performs $\theta\left(G_{x} G_{y} G_{t} n\right)$ distance tests and computes $\theta\left(n H_{s}^{2} H_{t}\right)$ densities. Since $H_{s}$ is smaller than $G_{x}$ and $G_{y}$ and since $H_{t}$ is smaller than $G_{t}$, the complexity of the algorithm is $\theta\left(G_{x} G_{y} G_{t} n\right)$ and it requires $\theta\left(G_{x} G_{y} G_{t}\right)$ memory.

\section{Algorithm Design and Engineering}

\subsection{Point-based Algorithm}

The voxel-based algorithm suffers from a massive cost of computing distances. The gold implementation reduces the number of distance computation by decomposing the domain, but that still incurs many distance calculations. Most of these calculations are unnecessary since we know where each point will radiate density. The point-based algorithm $\mathrm{PB}$, given in Algorithm 2 leverages that property.

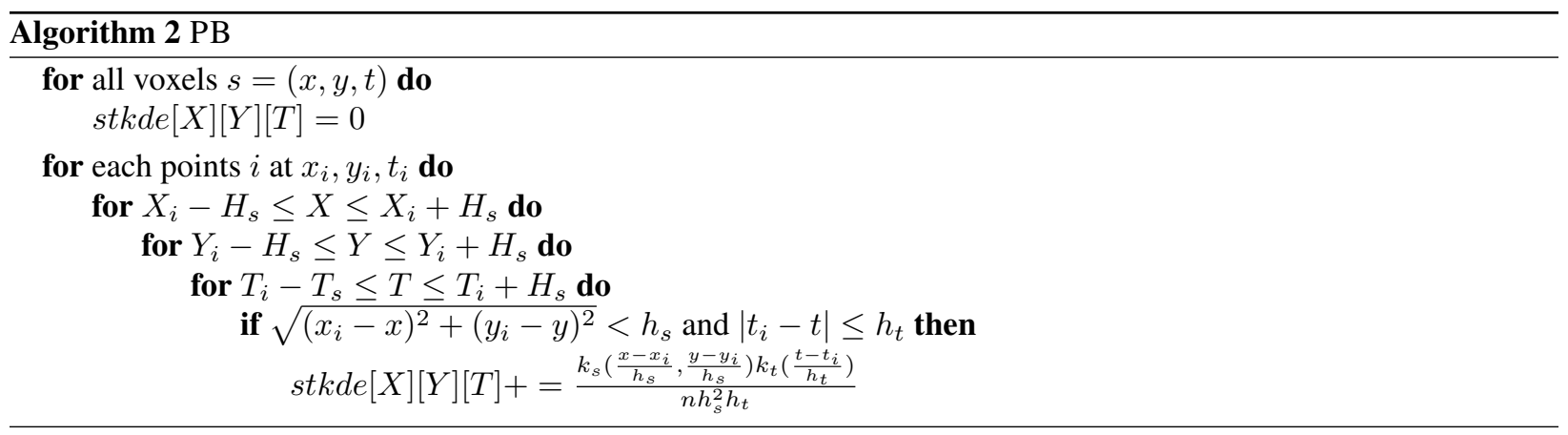

The algorithm PB incurs mostly two costs. Initializing the memory which costs $\Theta\left(G_{x} G_{y} G_{t}\right)$ and computing the impact of each point $\Theta\left(n H_{s}^{2} H_{t}\right)$. Note that it is possible that either of these two terms is significantly larger than the other. Therefore the complexity of the algorithm is $\Theta\left(G_{x} G_{y} G_{t}+n H_{s}^{2} H_{t}\right)$.

\subsection{Exploiting Symmetries}

When the bandwidth is large, computing the density contribution of each point each for voxel in the bandwidth is the most expensive operation. Each of these $n\left(2 H_{s}\right)^{2} 2 H_{t}$ calculations costs approximately 40 floating point operations. (The exact cost is difficult to estimate since there are floating point additions, multiplications, divisions, and square root operations).

One can remark that the calculation is mostly redundant since for one particular point, its contribution to the neighboring voxels can be decomposed in two components. $K_{s}(x, y)$ only depends on the spatial coordinate of the voxel with respect to the point and does not depend on its temporal coordinate. And $K_{t}(t)$ only depends on the temporal coordinate and not on the spatial coordinates. See Figure 3 for a graphical depiction.

Therefore, we write an algorithm to leverage these symmetries in the problem. We present three variants, the PB-DISK variant ensures that the invariant on the spatial domain is only computed once. The PB-BAR variant ensures that the invariant on the temporal domain is only computed once. The PB-SYM variant computes independently the 

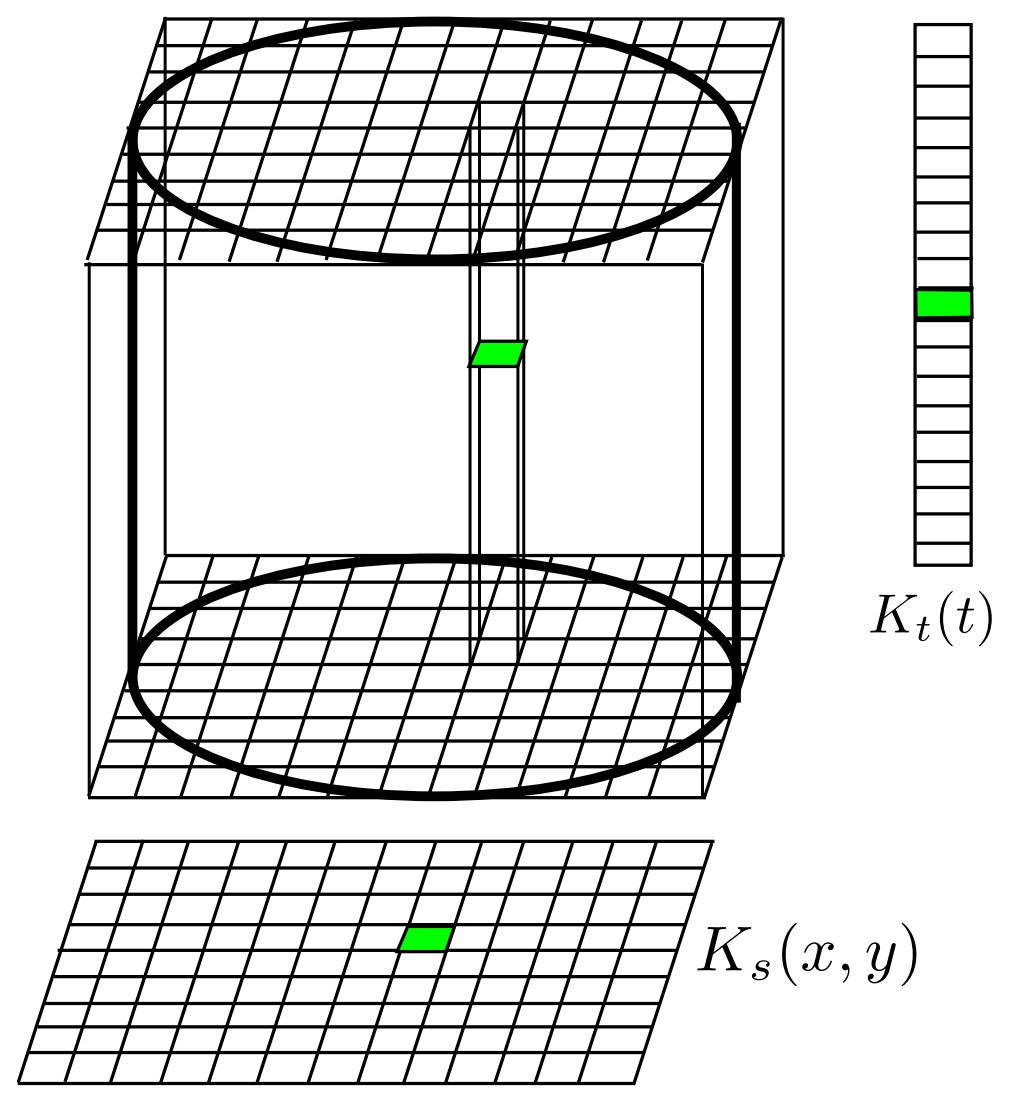

Figure 3: The contribution to the density estimate of each voxel of a cylinder can be decomposed in two terms, one temporally invariant $K_{s}(x, y)$ and one spatially invariant $K_{t}(t)$. 
spatial invariant and the temporal invariant and then adds the density caused by the point by taking the product of the two invariants. (See pseudocode in Algorithm 3) Notice that leveraging the symmetries in the calculation does not change the complexity (PB-SYM still has a complexity of $\Theta\left(G_{x} G_{y} G_{t}+n H_{s}^{2} H_{t}\right)$ ) but reduces the number of flops.

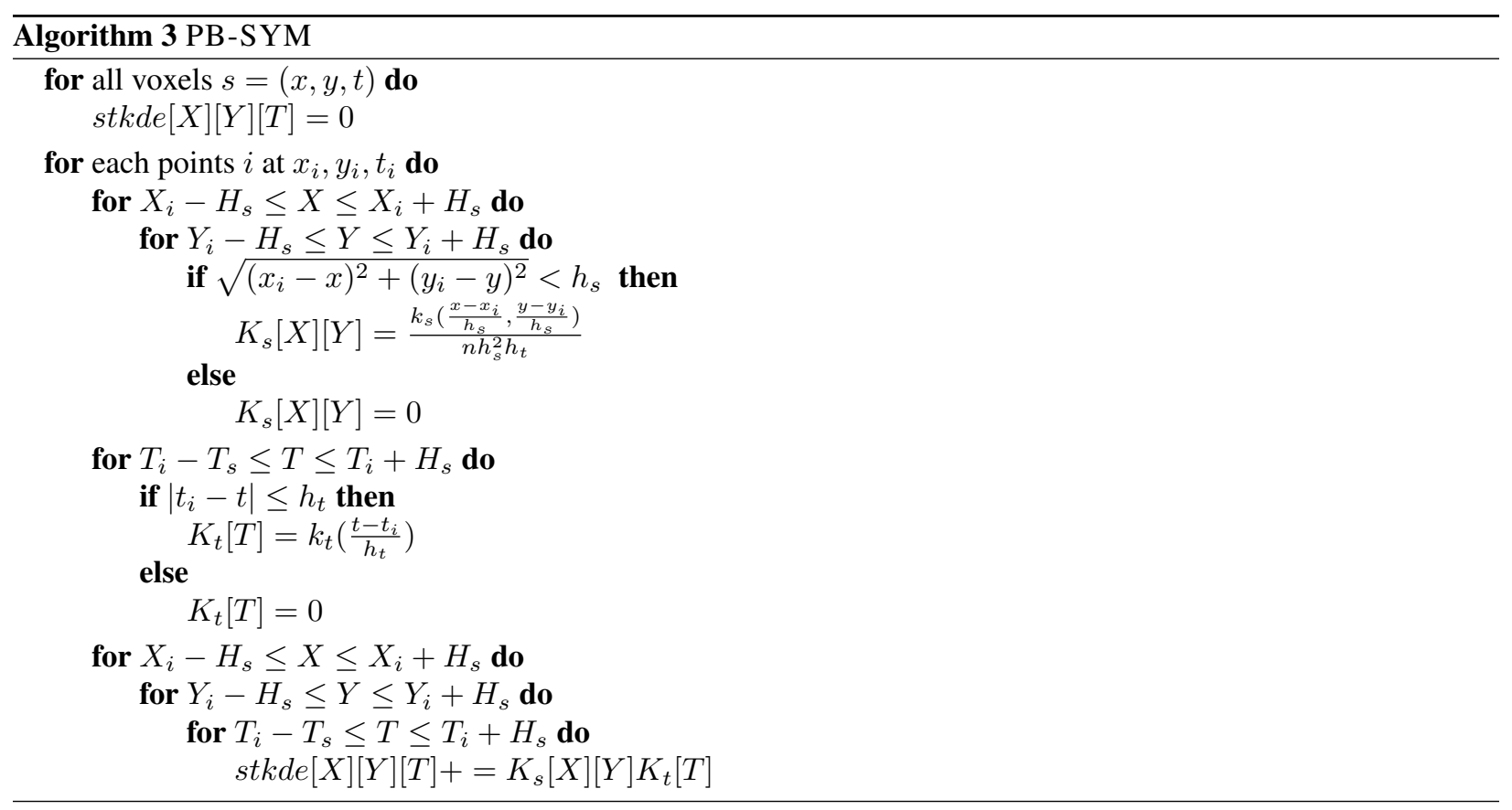

Notice that this exploitation of symmetries is not possible in a voxel-based algorithm.

\section{Domain-based parallelism}

\subsection{Domain Replication}

The simplest way to parallelize this kind of computation is to split the points equally among the $P$ different computational units. Though, if two close-by points are computed simultaneously, the cylinders of density around them might intersect and there is a race condition.

The PB-SYM-DR algorithm solves the data race issue by having each of the $P$ computational unit aggregate the result on a local copy of the domain. Once all the points have been processed, the $P$ copies need to be summed. The pseudo code of PB-SYM-DR is given in Algorithm 4

This algorithm has a memory requirement of $\Theta\left(P G_{x} G_{y} G_{t}\right)$ and a parallel amount of work of $\Theta\left(P G_{x} G_{y} G_{t}+\right.$ $n H_{s}^{2} H_{t}$ ). Fortunately, the increase in work enables the computation to be pleasingly parallel. (There are actually in three pleasingly parallel phases: initializing the memory, processing the points, and reducing the partial results.)

\subsection{Domain Decomposition}

Another strategy to avoid the data race is to compute voxel intensity independently for different subdomains. We call this method PB-SYM-DD (Algorithm 5). It splits the domain in AxBxC subdomains and each subdomain is associated the points which cylinder intersects with a voxel of the subdomain. Then the algorithm proceeds with handling each subdomain independently by only considering the impact of the data-point on voxels within the subdomain.

The main problem with Domain Decomposition is that it requires some points to be replicated across multiple subdomain which incurs additional work. Indeed, if a point is replicated in multiple subdomains, its cylinder is split across multiple subdomains as well. Assuming the split is temporal, then using PB-SYM in each subdomain 

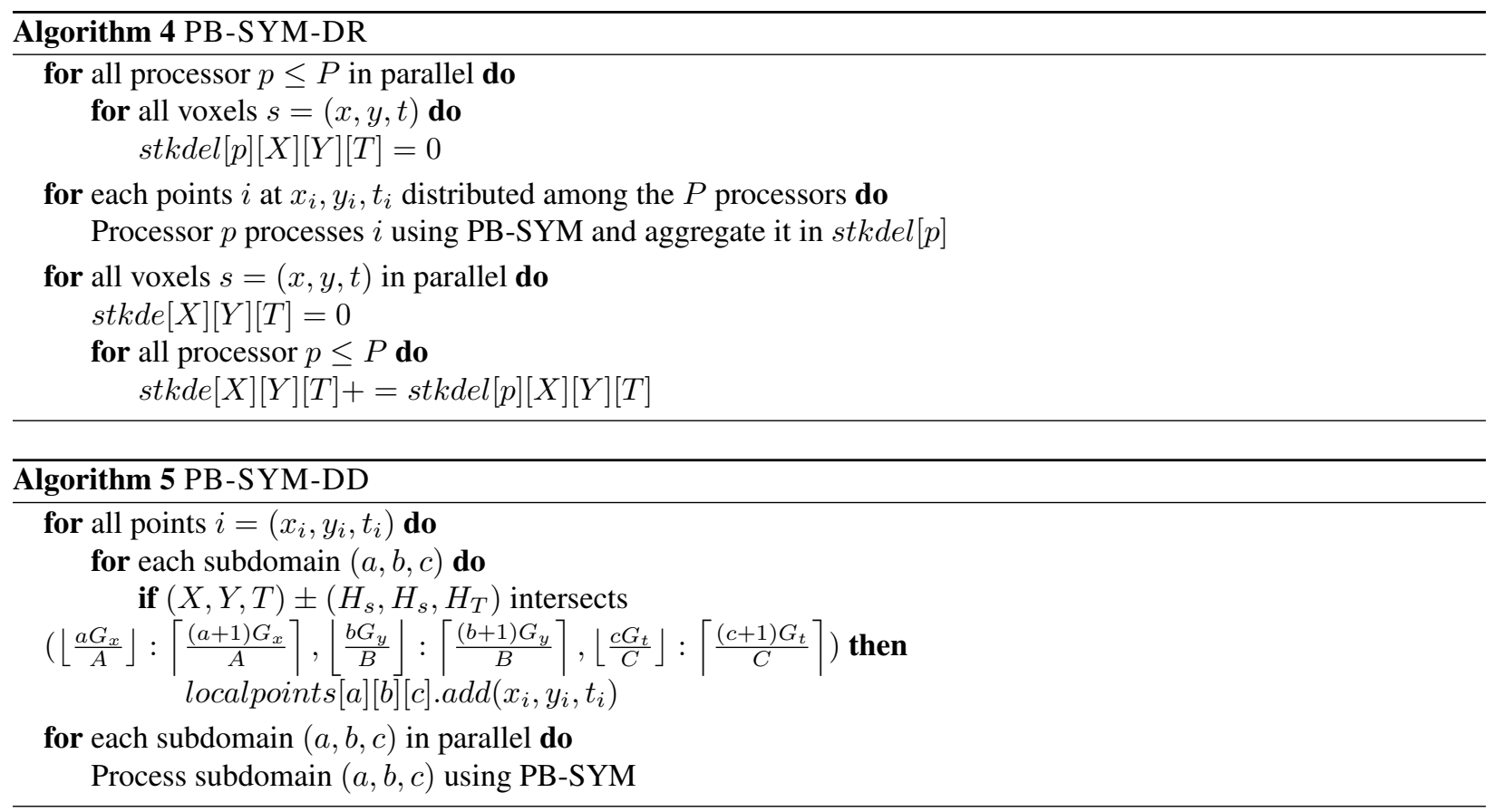

requires computing part of the temporal invariant, the entire spatial invariant, and part of the cylinder. Therefore both subdomains need to compute the entire spatial invariant. See Figure 4 for a graphical depiction of this phenomenon.

If the algorithms keeps the number of subdomains small such that each point is replicated less than a constant number of times, then the work expressed in Big-Oh notation does not change. (This can be achieved by having subdomains larger than $H_{s} \times H_{s} \times H_{t}$.) However, the practical amount of work can increase by a non-negligable factor.

Another issue with Domain Decomposition is load imbalance. The points are unlikely to be equally distributed in the domain space, but more likely clustered around some locations. The subdomain containing a cluster will have a significantly higher work than other subdomains. And this work is not executed in parallel. One could decompose the domain more, but replicated point will incur an even higher overhead which may end up nullifying the gain in load balance.

\section{Point-based parallelism}

\subsection{Point Decomposition}

Both of the previous strategies increase the amount of work significantly. We want to achieve parallelism without cutting a cylinder and without requiring each thread to have its own memory space. To achieve that goal, we propose the PB-SYM-PD algorithm that decomposes the points in sets that can be safely performed simultaneously.

As long as two points are separated by $2 h_{s}$ in space dimension or by $2 h_{t}$ in time, the cylinders induced by the two points will not overlap. PB-SYM-PD decomposes the points in $A \times B \times C$ subdomain of identical size. As long as these subdomains are larger than $2 h_{s}$ in a spatial dimension and $2 h_{t}$ in the temporal dimension, the points in domain $(x, y, z)$ can be done concurently with the points in $(x+2, y, z)$. See Figure 5 for a graphical depiction.

We implemented the first version of this algorithm that organizes the subdomain in 8 sets where the first set is composed of the subdomains $(2 i, 2 j, 2 k)$, the second of all the subdomains $(2 i+1,2 j, 2 k)$, the third of all the subdomains $(2 i, 2 j+1,2 k)$, etc.. The algorithm processes the sets the one after the other by doing each subdomain within a set in parallel. Two such sets are depicted in Figure 5. In practice, this is implemented using 8 OpenMP parallel-for constructs. 


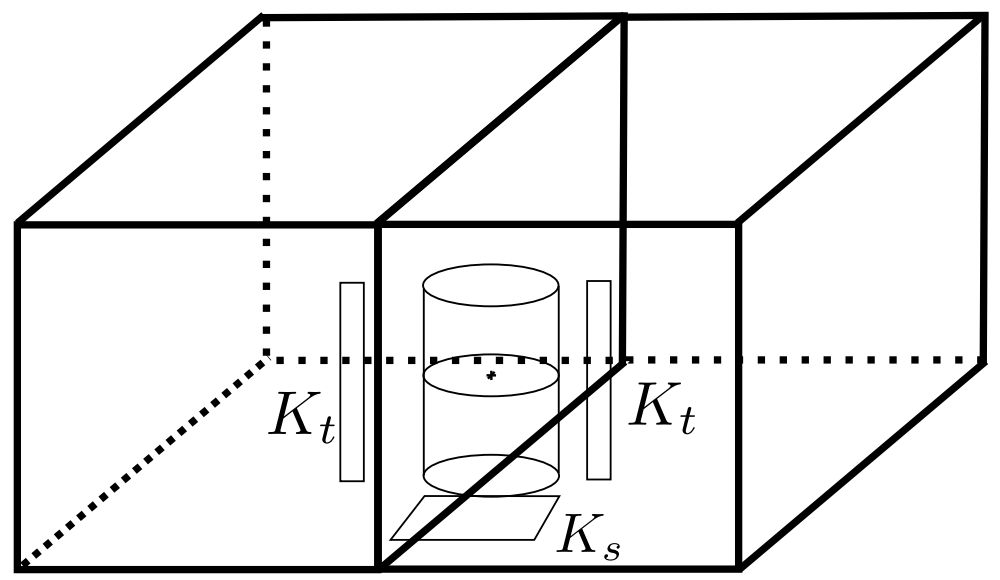

Figure 4: When a cylinder is split among two subdomains, PB-SYM-DD causes an overhead because one of the invariant needs to be recomputed. Here each subdomains compute part of the temporal invariant $K_{s}$, but both need to compute the spatial invariant $K_{t}$.

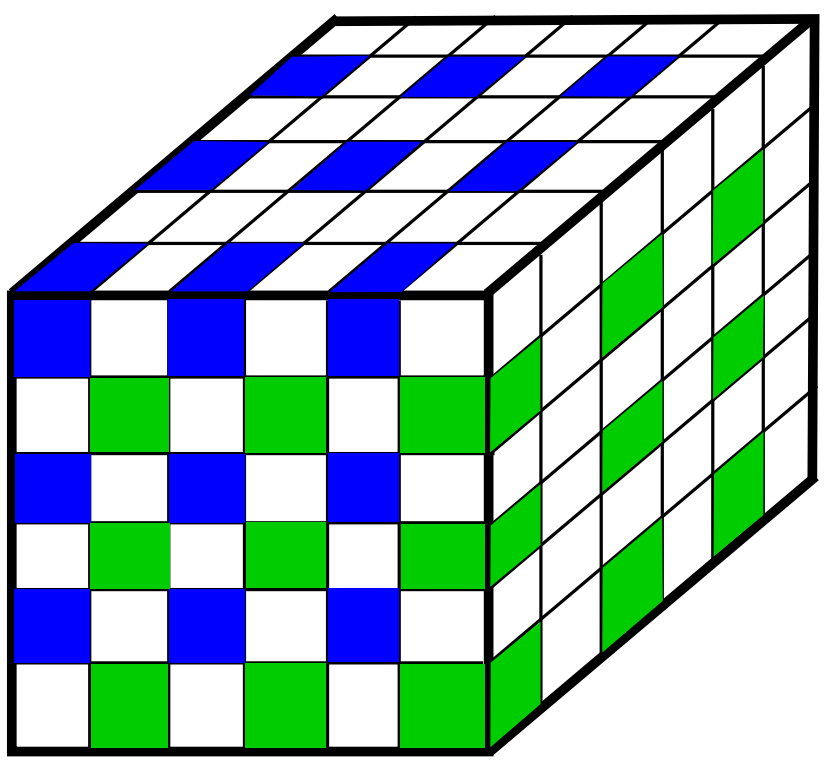

Figure 5: Provided the subdomains are larger than twice the bandwidth, the points contained in each blue boxes can be performed simultaneously. 

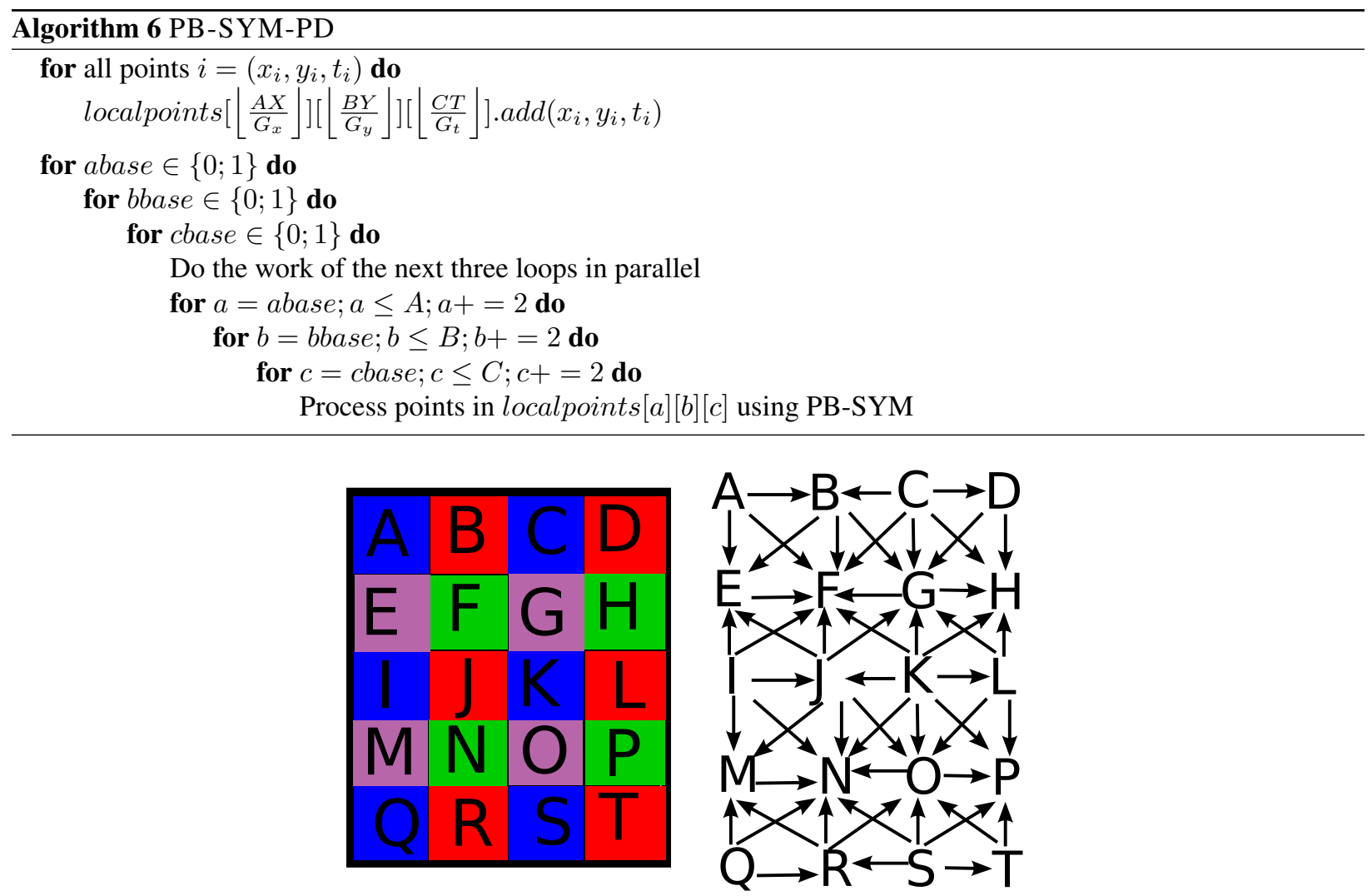

Figure 6: Dependency structure implied by a coloring of the subdomain. (Shown for a 2D decomposition for simplicity.)

\subsection{Coloring and Scheduling}

Note that such an implementation overconstrains the execution of the algorithm. Indeed, despite they are not in the same set, subdomain $(1,0,0)$ and $(64,64,64)$ can be computed at the same time. The real constraint is that the points contained in a subdomain can be processed safely as long as none of the point contained in a neighboring subdomain is being processed. Using the OpenMP tasking construct with dependency introduced in OpenMP 4.0 [Boa13], one can precisely express this constraint, which is a 27-point stencil constraint.

Formally speaking, this problem is a combination of multiple very classical graphs and scheduling problem. Modeling the subdomains as a 27-point stencil graph, identifying sets of subdomains to perform in parallel is coloring the vertices of the graph so that no two neighboring vertices share the same color. It differs from classical graph coloring problems (surveyed in [GMP05]) in that the objective is not to minimize the number of colors or to balance the number of vertices of each color but to minimize the execution time of the implied schedule.

The implied schedule is actually a scheduling of a graph of dependency which is extracted from the coloring. Each edge of the stencil graph is oriented from the vertex of the lowest color to the vertex with the highest color. Each vertex is associated with a processing time proportional to the number of points inside the sub-domain the vertex represents and the neighboring subdomains. This construction is represented in Figure 6

The OpenMP scheduler is not explicit in which order tasks that are available are performed. Though it is reasonable to assume that the tasks will be scheduled using a greedy algorithm. Therefore, the classic guarantee from Graham's List Scheduling [Gra69] is valid:

$$
T_{P} \leq \frac{T_{1}-T_{\infty}}{P}+T_{\infty}
$$

where $T_{1}$ is the total amount of work to perform and $T_{\infty}$ is the length of the longest chain in the dependency graph. 
We propose to reduce the critical path by using a load-aware coloring of the subdomains. We use a greedy coloring algorithm which considers the subdomains in a particular order and for each subdomain will color it with the smallest color that does not conflict with the already colored neighbors. Such greedy coloring algorithm are commonly used in parallel computing applications [GMP05, $\mathrm{BBÇ}^{+} 05$, DBDR16]. However, we will use an ordering that color the vertices in non-increasing order of the number of points contained in the subdomain. While this algorithm is a heuristic, it is likely to obtain a coloring that minimizes the critical path 1 . We call this algorithm to compute STKDE: PB-SYMPD-SCHED.

To decrease the critical path further, we propose an other algorithm PB-SYM-PD-REP which replicates the subdomains that are on the critical path (and its neighbors) of the graph so that the points of a subdomain can be performed in parallel. As long as the critical path is longer than $\frac{n}{2 P}$, the tasks on the path are replicated an additional time and the critical path is recomputed. Replication of subdomain causes to have to initialize additional subdomains and to reduce them which increases the work in a similar way PB-SYM-DR. However, the replication should be limited to a few chains in the graph and therefore should limit the overhead. Note that this problem is similar to scheduling a DAG of moldable tasks [LTW02, Hun13].

\section{Experiments}

\subsection{Experimental Setting}

Execution environment The machine used to perform the experiments is composed of two Intel Xeon E5-2667 v3 processors for a total of 16 cores clocked at $3.2 \mathrm{Ghz}$. Despite each core is capable of hosting 2 hyperthreads, hyperthreading was disabled. The machine is equipped with 128GB of DDR4 clocked at $2.133 \mathrm{Ghz}$. The node uses RHEL 7.2 and Linux 3.10. All codes are written in C++ and are compiled using GCC 5.3, which implements OpenMP 4.0, with optimization flags -O3 - march=native -fopenmp -DNDEBUG. All reported execution times exclude all IOs.

Dataset Four datasets are used in our experiments. Their key attributes are summarized in Table 2 Out first dataset, Dengue, is of epidemiological nature and consists of the space-time locations of dengue fever cases reported to the Sistema de Vigilancia en Salud Pública for the city of Cali, Colombia during 2010 and 2011. The system is updated on a daily basis with records of individuals that have been diagnosed with dengue fever, including patient address and the date of diagnosis. Addresses are standardized to a common address format, and spelling and other syntactical errors are manually corrected [DCRV13]; they are then geocoded and masked to the nearest street intersection level to maintain privacy [KCS04]. In 2010, 9606 were successfully geocoded (11,760 reported cases, or $81.7 \%$ ), whereas in 2011, 1562 cases were successfully geocoded (1822 reported cases, or $85.7 \%$ ).

The PollenUS dataset is composed of 588K tweets of US users from February 2016 to April 2016 related to Pollen (i.e., that mention keywords such as "pollen" and "allergy"). Tweets that did not contain a precise localization were approximated by picking a random location in the approximated region provided by Gnir 2 Tweets are mostly located in the contiguous continental US. Therefore, this is the region we modeled with a spatial resolution ranging from $.2^{\circ}$ to $1^{\circ}$ and a temporal resolution of 1 day.

The Flu dataset was obtained from the Animal Surveillance database of the Influenza Research Database 3 , The dataset contains observations of birds tested positive for carrying any strain the avian flu from 2001 to 2016 . The birds were observed all over the world and the modeled region spans as West as Alaska and East as Japan, as South as the southern shore of Australia and as North as the Northern shore of Russia. The domain is modeled with spatial resolution from $.2^{\circ}$ to $1^{\circ}$ and a temporal resolution of 1 day.

The eBird ${ }^{4}$ dataset is a collection of worldwide rare bird spotting obtained from a crowdsourcing effort managed by Cornell's Lab or Ornithology [CLoO16]. Despite the service was launched in 2002, the database contains many

\footnotetext{
${ }^{1}$ The complexity of this problem is currently unknown to the authors. The general case of an arbitrary graph is NP-Hard by reduction to graph coloring. But the case of a 1D stencil is clearly polynomial.

2 http://www.gnip.com/

3 https://www.fludb.org/

4 http://ebird.org
} 


\begin{tabular}{|l|rrr|rr|}
\hline Instance & $\mathrm{n}$ & $G_{x} \times G_{y} \times G_{t}$ & Size & $H_{s}$ & $H_{t}$ \\
\hline Dengue_Lr-Lb & 11056 & $148 \times 194 \times 728$ & $79 \mathrm{MB}$ & 3 & 1 \\
Dengue_Lr-Hb & 11056 & $148 \times 194 \times 728$ & $79 \mathrm{MB}$ & 25 & 1 \\
Dengue_Hr-Lb & 11056 & $294 \times 386 \times 728$ & $315 \mathrm{MB}$ & 2 & 1 \\
Dengue_Hr-Hb & 11056 & $294 \times 386 \times 728$ & $315 \mathrm{MB}$ & 50 & 1 \\
Dengue_Hr-VHb & 11056 & $294 \times 386 \times 728$ & $315 \mathrm{MB}$ & 50 & 14 \\
PollenUS_Lr-Lb & 588189 & $131 \times 61 \times 84$ & $2 \mathrm{MB}$ & 2 & 3 \\
PollenUS_Hr-Lb & 588189 & $651 \times 301 \times 84$ & $62 \mathrm{MB}$ & 10 & 3 \\
PollenUS_Hr-Mb & 588189 & $651 \times 301 \times 84$ & $62 \mathrm{MB}$ & 25 & 7 \\
PollenUS_Hr-Hb & 588189 & $651 \times 301 \times 84$ & $62 \mathrm{MB}$ & 50 & 14 \\
PollenUS_VHr-Lb & 588189 & $6501 \times 3001 \times 84$ & $6252 \mathrm{MB}$ & 100 & 3 \\
PollenUS_VHr-VLb & 588189 & $6501 \times 3001 \times 84$ & $6252 \mathrm{MB}$ & 50 & 3 \\
Flu_Lr-Lb & 31478 & $117 \times 308 \times 851$ & $117 \mathrm{MB}$ & 1 & 1 \\
Flu_Lr-Hb & 31478 & $117 \times 308 \times 851$ & $117 \mathrm{MB}$ & 2 & 3 \\
Flu_Mr-Lb & 31478 & $233 \times 615 \times 1985$ & $1085 \mathrm{MB}$ & 2 & 3 \\
Flu_Mr-Hb & 31478 & $233 \times 615 \times 1985$ & $1085 \mathrm{MB}$ & 4 & 7 \\
Flu_Hr-Lb & 31478 & $581 \times 1536 \times 5951$ & $20260 \mathrm{MB}$ & 5 & 7 \\
Flu_Hr-Hb & 31478 & $581 \times 1536 \times 5951$ & $20260 \mathrm{MB}$ & 10 & 21 \\
eBird_Lr-Lb & 291990435 & $357 \times 721 \times 2435$ & $2391 \mathrm{MB}$ & 2 & 3 \\
eBird_Lr-Hb & 291990435 & $357 \times 721 \times 2435$ & $2391 \mathrm{MB}$ & 6 & 5 \\
eBird_Hr-Lb & 291990435 & $1781 \times 3601 \times 2435$ & $59570 \mathrm{MB}$ & 10 & 3 \\
eBird_Hr-Hb & 291990435 & $1781 \times 3601 \times 2435$ & $59570 \mathrm{MB}$ & 30 & 5 \\
\hline
\end{tabular}

Table 2: Properties of the datasets. The domain is expressed in voxels and in MB. Bandwidth are expressed in voxels.

older entries of birds that are known to have been observed at the time. For our experiment, we use the last 20 years of bird spotting and model the entire world with a temporal resolution of 3 days, and a spatial resolution of either $.1^{\circ}$ or . $5^{\circ}$.

For all datasets, we created multiple instances of the problem coded $\mathrm{Lr}, \mathrm{Mr}, \mathrm{Hr}$ for low, medium, and high resolution and $\mathrm{Lb}, \mathrm{Hb}, \mathrm{VHb}$ for low, high and very high bandwidth. See details in Table 2

\subsection{Algorithm design}

Table 3 presents the runtime of the point-based algorithms presented in Section 3 The runtime are given in seconds. As expected for each dataset, the runtime increases with resolution and bandwidth. Exploiting the disk invariant with PB-DISK provides a large reduction in the runtime, even more so when the temporal bandwidth is high. PB-BAR provides a more modest time reduction.

PB-SYM combines the reductions of both PB-BAR and PB-DISK and achieves the best performance in most cases. PB-SYM provides improvements up to a factor of 6.969 on the PollenUS_Hr-Hb case. The cases where PBSYM provides little improvement are explained either by the instance having a low bandwidth, and therefore there is little redundant computation to take advantange of, or by the fact that the runtime is dominated by initialization cost. The breakdown of the runtime of PB-SYM is given in Figure 7 and shows that PB-SYM is composed of two phases, 


\begin{tabular}{|l|rr|rrrr|r|}
\hline & \multicolumn{7}{|c}{ Time (in seconds) } \\
Instance & VB & VB-DEC & PB & PB-DISK & PB-BAR & PB-SYM & PB-SYM \\
\hline Dengue_Lr-Lb & 219.163 & 2.283 & 0.040 & 0.029 & 0.035 & 0.028 & 1.429 \\
Dengue_Lr-Hb & 220.591 & 13.878 & 1.298 & 0.564 & 1.152 & 0.499 & 2.601 \\
Dengue_Hr-Lb & 866.445 & 9.522 & 0.089 & 0.082 & 0.085 & 0.084 & 1.060 \\
Dengue_Hr-Hb & 871.774 & 55.206 & 5.169 & 2.272 & 4.563 & 2.074 & 2.492 \\
Dengue_Hr-VHb & 1056.172 & 404.845 & 51.885 & 11.478 & 42.994 & 7.431 & 6.982 \\
PollenUS_Lr-Lb & 518.859 & 7.639 & 1.106 & 0.347 & 0.922 & 0.256 & 4.320 \\
PollenUS_Hr-Lb & 12721.001 & 189.337 & 23.539 & 7.700 & 18.527 & 4.708 & 5.000 \\
PollenUS_Hr-Mb & 17179.482 & 3126.947 & 357.743 & 86.129 & 295.791 & 57.528 & 6.219 \\
PollenUS_Hr-Hb & & & 2666.104 & 583.175 & 2212.626 & 382.566 & 6.969 \\
PollenUS_VHr-Lb & & & 2428.126 & 1004.174 & 1949.988 & 759.722 & 3.196 \\
PollenUS_VHr-VLb & & & 603.789 & 240.236 & 488.388 & 179.834 & 3.357 \\
Flu_Lr-Lb & 926.360 & 3.691 & 0.035 & 0.032 & 0.034 & 0.032 & 1.094 \\
Flu_Lr-Hb & 966.328 & 3.797 & 0.081 & 0.046 & 0.070 & 0.042 & 1.929 \\
Flu_Mr-Lb & 8591.165 & 30.355 & 0.305 & 0.278 & 0.298 & 0.277 & 1.101 \\
Flu_Mr-Hb & 8957.175 & 32.018 & 0.714 & 0.384 & 0.608 & 0.323 & 2.211 \\
Flu_Hr-Lb & & 536.091 & 5.702 & 5.089 & 5.454 & 5.059 & 1.127 \\
Flu_Hr-Hb & & 591.955 & 12.795 & 6.822 & 10.992 & 7.072 & 1.809 \\
eBird_Lr-Lb & & & 396.811 & 147.951 & 322.580 & 125.248 & 3.168 \\
eBird_Lr-Hb & & & 6969.187 & 1897.051 & 5611.158 & 1067.395 & 6.529 \\
eBird_Hr-Lb & & & 8373.273 & 3226.016 & 6470.764 & 2229.460 & 3.756 \\
eBird_Hr-Hb & & & & & & 34577.745 & \\
\hline
\end{tabular}

Table 3: Runtime of different algorithm. The speedup of PB-SYM over PB is given. 


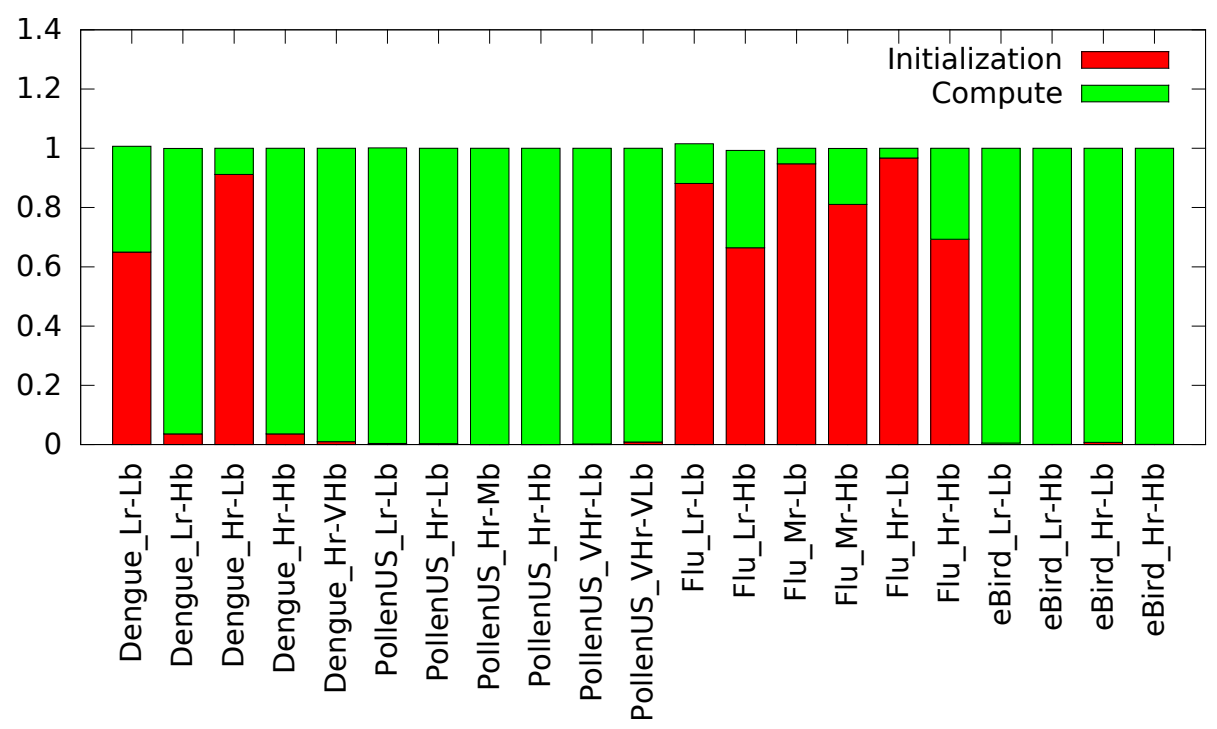

Figure 7: Breakdown of the runtime of PB-SYM. Some instances are mostly Initialization.

initializing the memory to store the density estimation of the voxels, and computing the density cylinder surrounding the points. PB-SYM reduces the computation cost but initializes the memory in the same way as PB. Initialization is a predominant runtime cost in the Flu dataset since there were only $31 \mathrm{~K}$ occurence of confirmed avian flu recorded in the last 15 years but they span most of the earth.

Comparing to the gold implementation would not be fair because it is in a different programming language. Though we implemented the VB algorithm, and also a variant, VB-DEC, that partitions the points in blocks of size equal to the bandwidth to only compute distances between voxels and points that have a chance to have an impact on it. The results are given in Table 3 and show that even VB-DEC is usually at least an order of magnitude larger than PB and typically two orders of magnitude larger than PB-SYM.

\subsection{Domain-based parallelism}

PB-SYM-DR The performance of the PB-SYM-DR algorithm is given in Figure 8. Because it replicates the domain space, PB-SYM-DR was not able to complete the calculations for Flu_Hr-Lb and Flu_Hr-Hb when using 8 threads and 16 threads as the memory requirement exceeds the 128GB available on the machine. None of the high resolution eBird instances could have their domain replicated.

All the instances that have a high initialization cost get a speedup lesser than 1 since the threads spend their time allocating extra memory and reducing the content of that extra memory. The only instances to achieve a speedup higher than 8 when using 16 threads are 3 of the PollenUS instances and one low resolution eBird instances. Not surprisingly, they are the instances with the largest fraction of computation. (See Figure 7 for reference.)

PB-SYM-DD The PB-SYM-DD algorithm (described in Section 4.2) also has some overhead induced by cut cylinder surrounding a point. To measure the overhead beforehand, we run the decomposition algorithm using different number of subdomains ranging from a single subdomain $(1 \times 1 \times 1)$ to a very fine grain decomposition in $64 \times 64 \times 64$ subdomains (except on the eBird_Hr_Hb where such a test is computationally expensive). The results of that experiment is presented in Figure 9. The figure presents the overhead of the decomposition relatively to PB-SYM. Two things are apparent. Some decomposition can actually reduce the runtime by providing better cache locality; for instance Flu_Hr-Lb is $9.8 \%$ faster using a 16x16x16 decomposition than PB-SYM. 


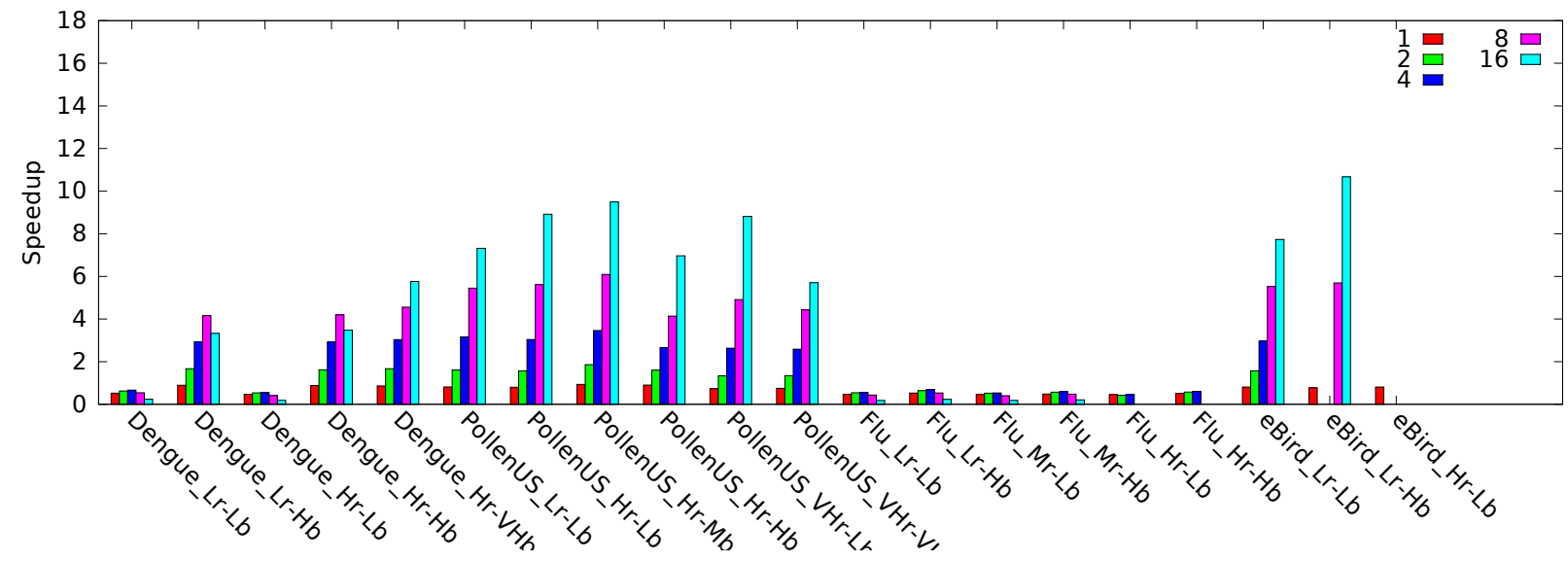

Figure 8: Speedup of PB-SYM-DR for different number of threads used. Some instances run out of memory.

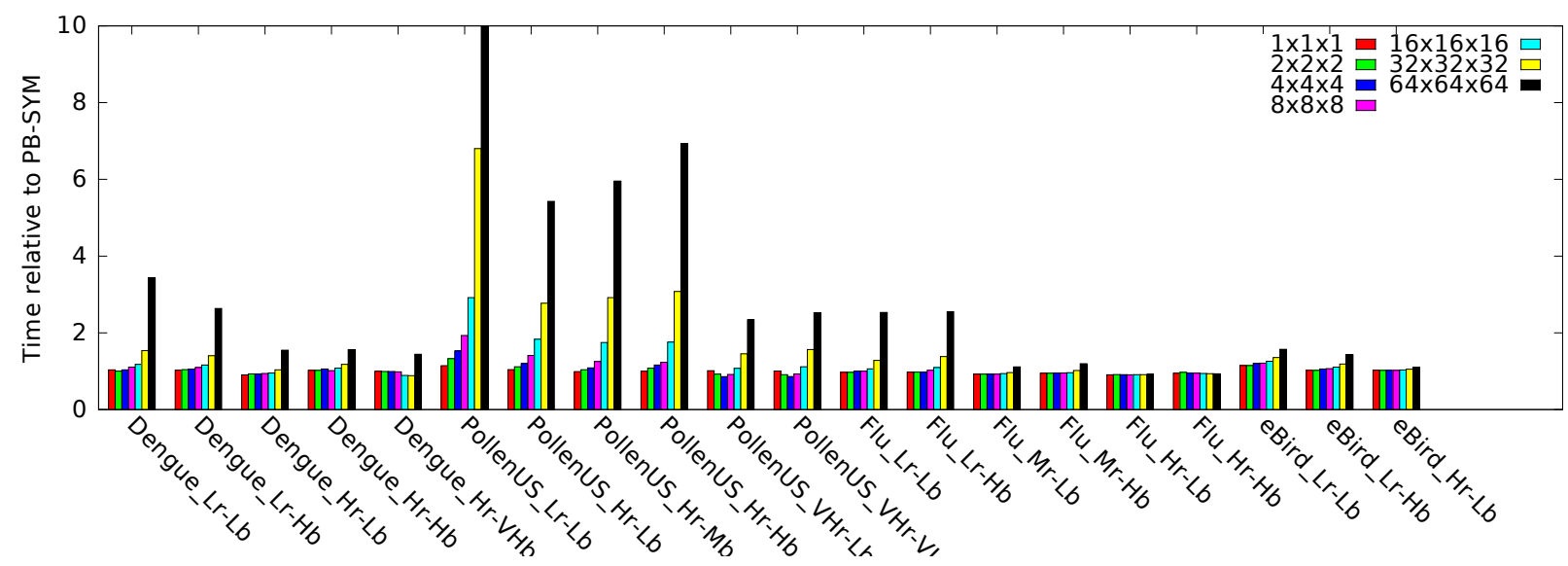

Figure 9: Overhead of PB-SYM-DD: runtime of PB-SYM-DD with a single thread normalized to PB-SYM. Some decomposition improve performance dues to better cache fitting. Overdecomposition can cause significant overhead.

However, in most cases, the decomposition actually increases the runtime. For instance, a 16x16x16 decomposition increases the amount of work by $9 \%$ in the Flu-Lr-Hb cases, and a 64x64x64 decomposition induces an overhead of $254 \%$. Even on the Dengue_Lr-Hb, a simple 4x4x4 decomposition already induces a 5\% overhead which grows to 69\% for a 16x16x16 decomposition. Such large decomposition are likely to be necessary since the points in the datasets are often organized in clusters: a decomposition in 16 subdomains is unlikely to provide a load balanced execution. The PollenUS suffers from the highest overheads, with a 16x16x16 decomposition leading to a $74 \%$ overhead on PollenUS-Hr-Mb, and a $495 \%$ overhead for a $64 \times 64 \times 64$ decomposition

The speedup achieved by a parallel execution of PB-SYM-DD using 16 threads is presented in Figure 10 This algorithm achieves overall better speedup than PB-SYM-DR. It achieves a speedup greater than 8 on 9 instances. Let us note that a speedup of 14.9 is achieved on Dengue_Hr-VHb using a 16x16x16 decomposition and of 14.8 on eBird_Hr-Hb with a $32 \times 32 \times 32$ decomposition.

Despite a significant overhead on the PollenUS_Hr instances, a speedup of 10 and 11.5 can be observed on the Mb and $\mathrm{Hb}$ cases using $8 \times 8 \times 8$ decomposition. This speedup is relatively impressive provided the decomposition induces an overhead of $25 \%$ and $23 \%$ in these two cases. The $8 \times 8 \times 8$ decomposition in that case is still imbalanced and a finer 


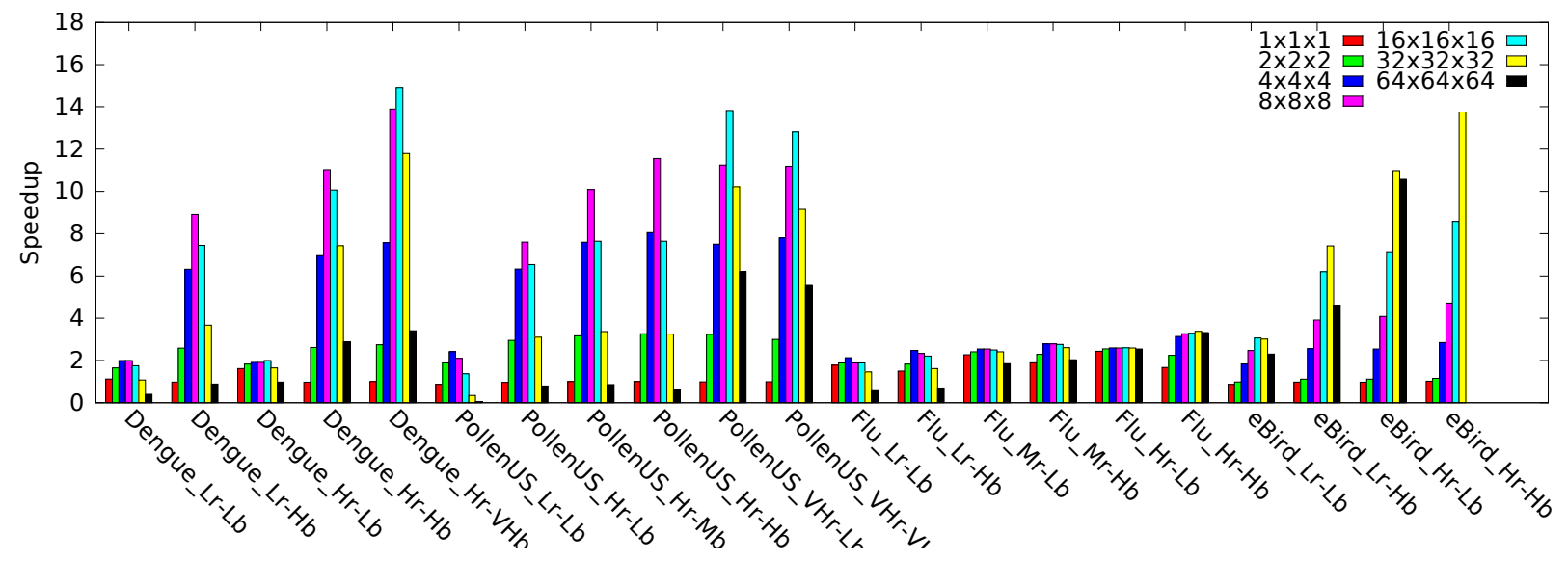

Figure 10: Speedup of PB-SYM-DD with 16 threads. While PB-SYM-DD achieves good speedup in some cases, the overdecomposition overhead usually hinders the performance.

decomposition can reach perfect load balance; however the overhead induced by the decomposition prevents taking advantages of that better load balance. For PB-SYM-DD to be useful, it is important to pick the decomposition to ensure load balance without suffering from an unbearable computational overhead.

A more modest speedup between 2 and 4 can be observed on the instances with higher initialization cost, such as on the Flu dataset. Indeed, most of the time is spent on initializing the memory to hold the density estimates. Even if the algorithm performs memory initialization in parallel, there is not much room for parallelism in memory operations. Even more so since the initialization touches the memory for the first time, which makes the operating system allocates the pages in physical memory. The speedup of the initialization phase using 16 threads is about the same for all instances and is at about 3 . The small speedup of PB-SYM-DD on instances with high initialization cost is completely explained by this phenomenon. Even if the compute phase was reduced to 0, the speedup on the entire calculation would only be 3.7 .

\subsection{Point-based parallelism}

The performance of PB-SYM-PD is shown in Figure 11 for different decomposition. Note that there is a requirement in PB-SYM-PD that the subdomain be larger than twice the bandwidth (see details in Section 5.1). Therefore if the number of subdomain leads to too small subdomains, the decomposition is adjusted to fit the required size.

PB-SYM-PD does not scale well on all instances. The highest speedup achieved on PollenUS_Lr-Lb is 2.6. It is interesting to notice that the speedup usually increases with decomposition size. However, the speedup is limited because of load imbalance. Figure 12 shows the implicit critical path within the PB-SYM-PD algorithm for the highest decomposition used in the experiment (64x64x64). Most instances have a critical path of about $10 \%$ of the total work. If Graham's bound is reached, that implies a speedup lesser than 6.15. PollenUS_Hr-Hb has a critical path of 55\% which implies a speedup lesser than 1.6. However, the speedup is typically lower because of the limited scalability in the initialization phase.

PB-SYM-PD-SCHED aims at improving the load balance by arranging the execution to process the most loaded subdomain first. Figure 12 shows that PB-SYM-PD-SCHED marginally decreases the critical path in all but one case. Even if that decrease is marginal, PB-SYM-PD-SCHED ensures that the highest loaded subdomain are executed first which tends to construct a better execution in practice. And one can see from Figure 13 that this scheduling improves parallelism significantly for the PollenUS instance. A superlinear speedup occurs on PollenUS_VHr-VLb: it is due to the decomposition of the points which improves the locality of the computation compared to the sequential execution.

However, the parallelism is still constrained by load imbalance. PB-SYM-PD-REP performs domain decomposition on the subdomain of the most loaded chains in the dependency graph until the critical is small. Figure 14 shows 


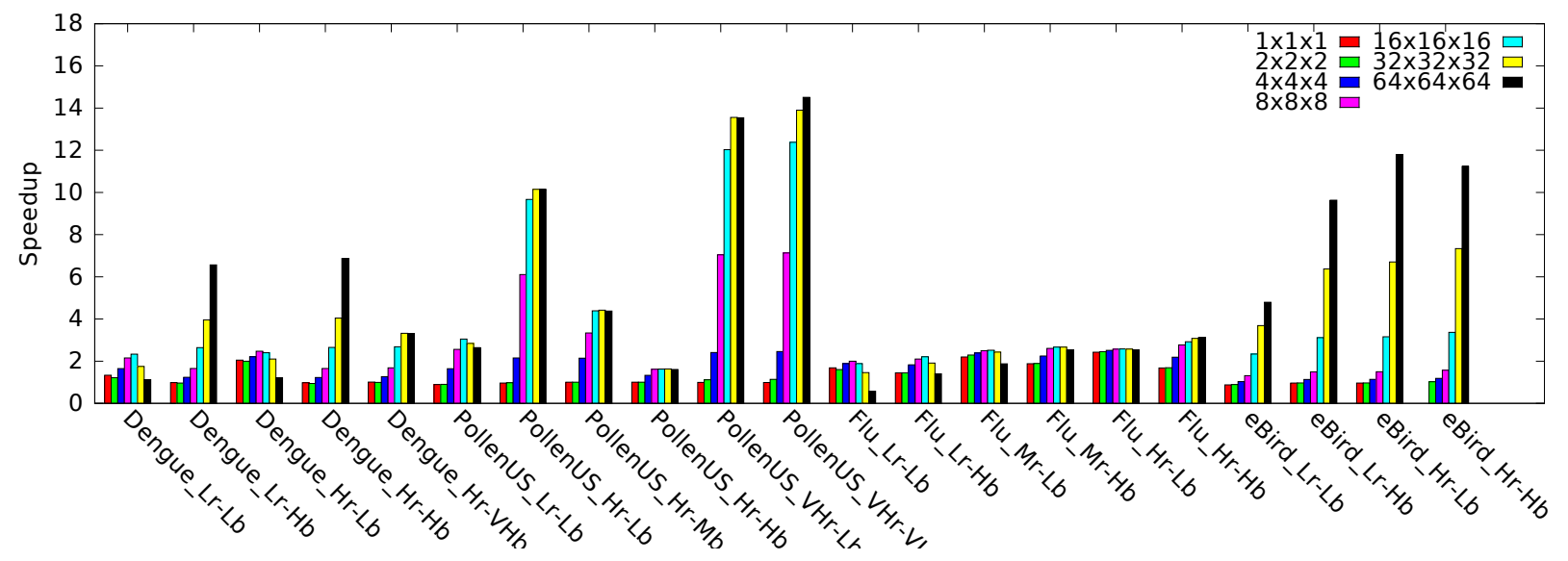

Figure 11: Speedup of PB-SYM-PD using 16 threads. Note that decompositions of subdomain smaller that twice the bandwidths are adjusted.

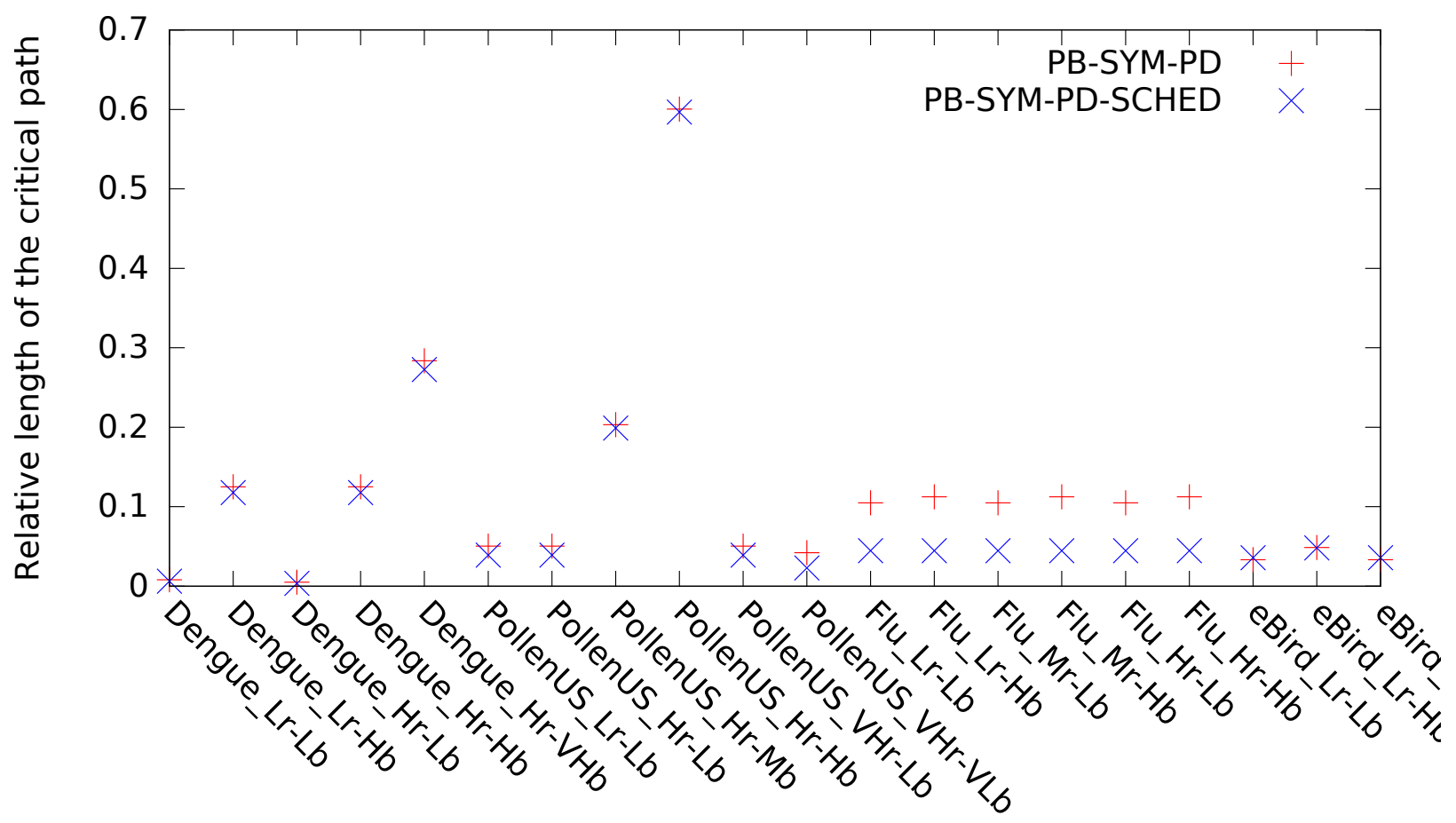

Figure 12: Length of the critical path of the parallel algorithms PB-SYM-PD and PB-SYM-PD-SCHED for a 64x64x64 decomposition 


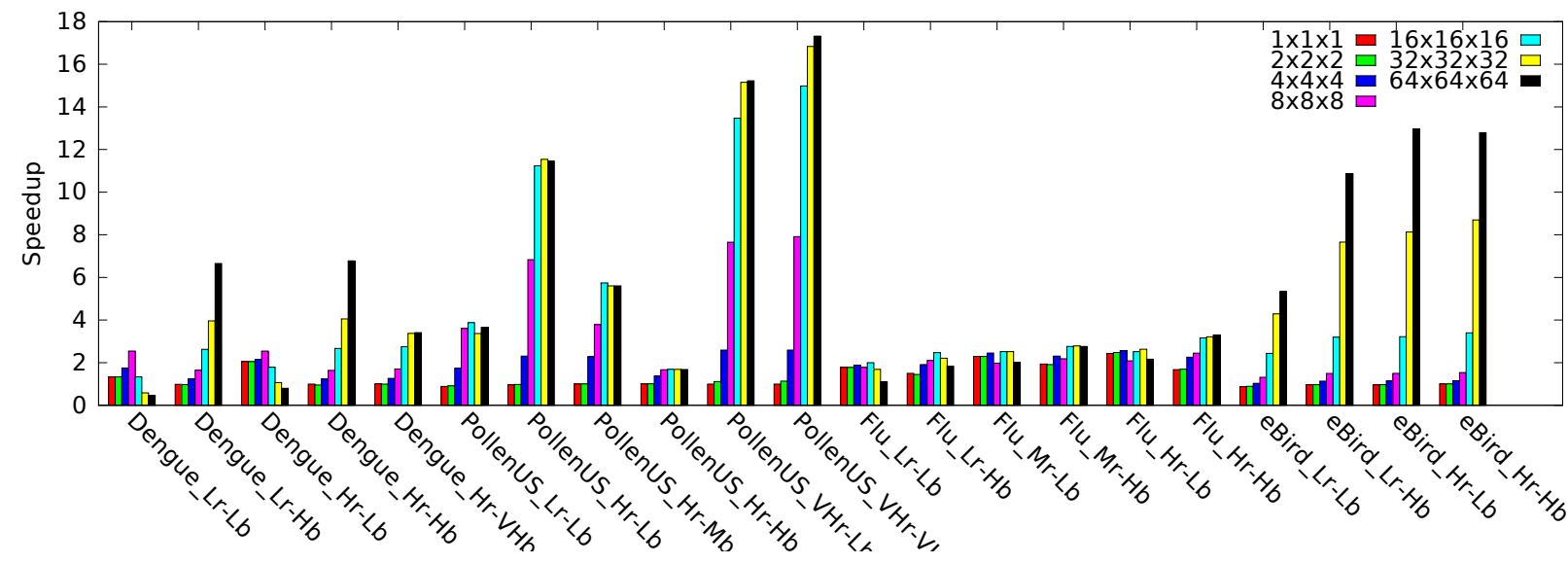

Figure 13: Speedup of PB-SYM-PD-SCHED with 16 threads.

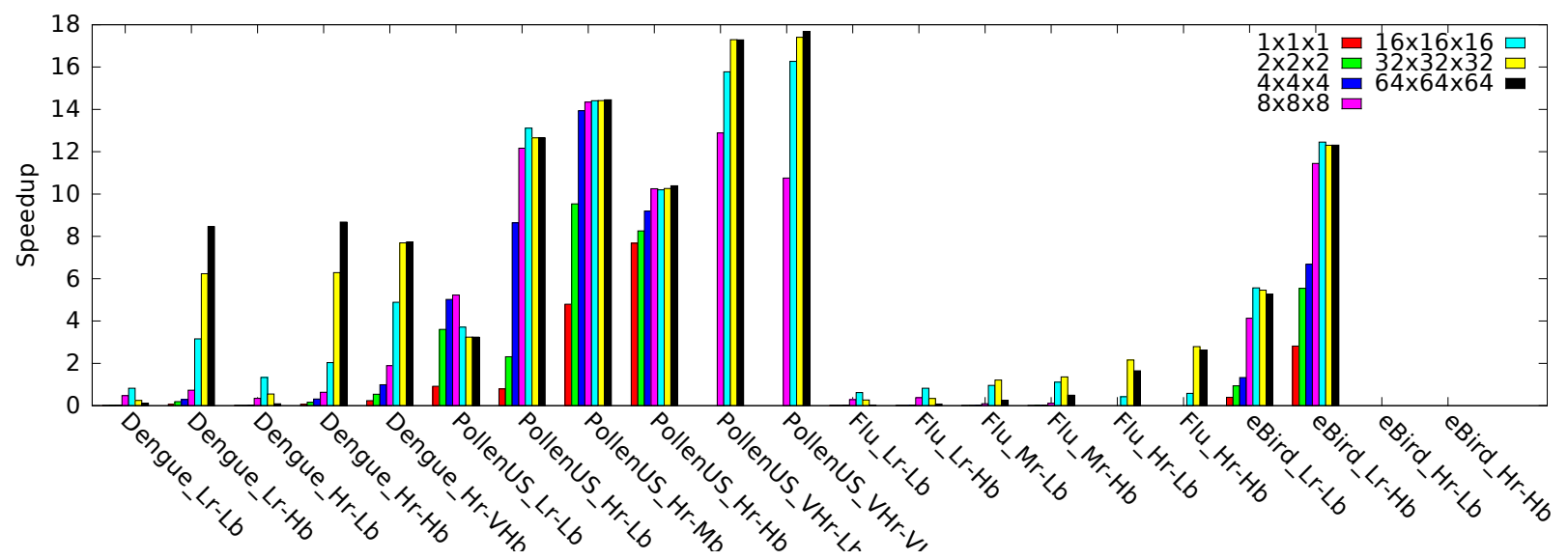

Figure 14: Speedup of PB-SYM-PD-REP using 16 threads for different decompositions. Flu_Hr-Lb and Flu_Hr-Hb run out of memory for small decomposition.

the speedup achieved by PB-SYM-PD-REP. Using PB-SYM-PD-REP achieves a speedup larger than 8 on 8 instances. Though the speedup is close to 0 for small decompositions and on certain instances. Indeed, when there is no (or little) decomposition, most of the domain is replicated and the execution has similar drawbacks as PB-SYM-DR.

\subsection{Summary and Discussion}

Figure 15 summarizes the performance of all our methods. It shows the performance achieved by the best configuration of each particular algorithm. On Dengue instances, PB-SYM-DD usually leads to the best performance because of its low overhead on that instance while maintaining a good load balance. On the PollenUS instances, the smart scheduling of PB-SYM-PD-SCHED-REP is necessary to reach the highest parallelism. The flu instances are mostly dominated to initialization overhead because it is very sparse, as such, PB-SYM-DR performs much worse than the other methods, and past that issue, the method leads to little performance difference. The eBird instances have a very small memory initialization overhead because they are dense in computation. This makes approaches that replicate the voxel space perform well at low resolution, but runs out of memory at high resolution there. 


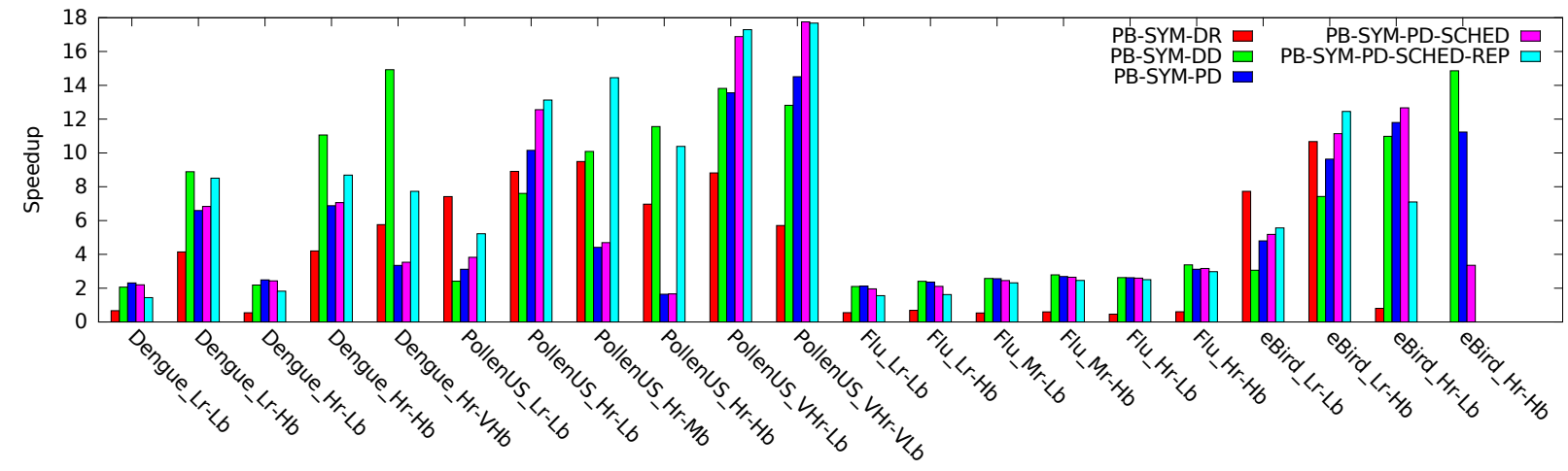

Figure 15: Best configurations

What we need to do is to develop a parametric model for the problem that will take into account memory availability, cost of memory initialization, expected cost of computing the kernel density. Using that model finding the best execution strategy becomes a combinatorial problem.

\section{Related Works}

The PhD Thesis of U. Lopez-Novoa [Lop15] is on the topic of computing kernel density estimation for arbitrarily shaped kernels. The crop and chop method presented revolves around cropping the voxel space around a point with similar goal as PB, and compute the density estimates in parallel on a multi-core CPU or on a GPU before the data is aggregated back on the CPU. The arbitrarily shaped density considered does not expose the invariants leveraged by PB-SYM which makes their work inapplicable to STKDE. [ECR17] considers the same variant of kernel density that we consider and expand the techniques of [Lop15] by considering coalescing. However, they still use a voxel based algorithm which is very slow (on a resolution lower than our lowest resolution, the parallel computation takes .6 seconds on a GPU which is slower than our reported sequential time on a larger instance).

The two most common spatial problems solved with parallel computing are N-body interactions [Aar03] and Particle in Cell simulations [KVKVO06]. STKDE is different in the sense that there is no iterating over the problem multiple times as we see in the timesteps of N-body or particle in cell, no direct interaction between points, and no field updates as in Particle in Cell (unless you consider a field discretized at a one voxel size which makes the analogy not quite useful).

The summation of kernel function, in particular of radial basis function attracted some attention [MXYB15, FLF11, YDGD03]. While some method presented in these papers are similar to some of the decomposition we discuss here, the mathematical property of the kernel function are different. In particular the kernel functions that these papers consider do not have the grid-aligned symmetries that the space-time kernel density estimate have. That symmetry is what we leverage in PB-SYM to gain an order of magnitude of acceleration and that leads to a more complex management of parallelism we investigated.

Spatial applications can use spatial partitioning techniques such as recursive bisection [BB87], jagged partition [USZS96, PA97, DRDÇ16], or rectilinear partition [Nic94, MS96]. Depending on the algorithms used for STKDE, the objective function is different. A partitioning for PB-SYM-DD needs a good load balance, to minimize the number of cut cylinders. But PB-SYM-PD needs a decomposition where the subdomains have a minimum size and the load balance critirion is harder to express since neighboring subdomains can not be processed simultaneously.

\section{Conclusion}

We presented in this paper the space-time kernel density estimation problem which is useful in the visualization of events located in space and time. We proposed sequential algorithms to decrease the complexity of the problem. And 
we investigated four parallel algorithms, two of which are pleasingly parallel but at the cost of not being work efficient. We then designed a work-efficient parallel algorithm but the dependency structure tends to prevent high degree of parallelism. Using graph coloring and moldable scheduling techniques we made that latter parallel algorithm more parallel by adding some work-overhead.

For each instance of the problem, one of the parallel algorithm achieved an interesting performance. However, it is clear that we need to model the instance and the platform to control the various overhead and be able to pick the parallel strategy that will derive the highest performance. It would also be interesting to look at distributed memory machines and accelerators to reduce the runtime further since real-time is desirable for interactive applications. In term of the application, we would like to investigate how these methods apply to a bandwidth that adapts to the density of population of the area is also of interest.

\section{Acknowledgment}

The authors would like to thank Dr. Daniel Janies for pointing us to the Flu dataset. Support from US NSF XSEDE Supercomputing Resource Allocation (SES170007) "Accelerating and enhancing multi-scale spatiotemporally explicit analysis and modeling of geospatial systems" is acknowledged. This material is based upon work supported by the National Science Foundation under Grant No. 1652442.

\section{References}

[Aar03] Sverre J. Aarseth. Gravitational N-Body Simulations: Tools and Algorithms. Cambridge University Press, 2003.

[BB87] Marsha Berger and Shahid Bokhari. A partitioning strategy for nonuniform problems on multiprocessors. IEEE TC, C36(5):570-580, 1987.

$\left[\mathrm{BBÇ}^{+}\right.$05] E.G. Boman, D. Bozdă̆, Ü.V. Çatalyürek, A.H. Gebremedhin, and F. Manne. A scalable parallel graph coloring algorithm for distributed memory computers. In Proc. of Euro-Par, pages 241-251, Aug 2005.

[Boa13] OpenMP Architecture Review Board. Openmp application program interface. Technical report, OpenMP Architecture Review Board, July 2013. Verion 4.0.0.

[CLoO16] New York Cornell Lab of Ornithology, Ithaca. ebird basic dataset. http://ebird.org/content/ebird/, May 2016. Version: EBD_relMay-2016.

[DBDR16] M. Deveci, E. G. Boman, K. D. Devine, and S. Rajamanickam. Parallel graph coloring for manycore architectures. In 2016 IEEE International Parallel and Distributed Processing Symposium (IPDPS), pages 892-901, May 2016.

[DCRV13] Eric Delmelle, Irene Casas, Jorge H Rojas, and Alejandro Varela. Spatio-temporal patterns of dengue fever in cali, colombia. International Journal of Applied Geospatial Research (IJAGR), 4(4):58-75, 2013.

$\left[\mathrm{DDC}^{+}{ }^{+}\right.$E] E. Delmelle, C. Dony, I. Casas, M. Jia, and W Tang. Visualizing the impact of space-time uncertainties on dengue fever patterns. International Journal of Geographical Information Science, 28(5):1107-1127, 2014.

[DRDÇ16] Mehmet Deveci, Sivasankaran Rajamanickam, Karen D. Devine, and Ümit V. Çatalyürek. Multi-jagged: A scalable parallel spatial partitioning algorithm. IEEE Trans. Parallel Distrib. Syst., 27(3):803-817, 2016.

[ECR17] Todd Eaglin, Isaac Cho, and William Ribarsky. Space-time kernel density estimation for real-time interactive visual analytics. In Proceedings of the 50th Hawaii International Conference on System Sciences, January 2017. 
[EE11] Lars Eisen and Rebecca J Eisen. Using geographic information systems and decision support systems for the prediction, prevention, and control of vector-borne diseases. Annual review of entomology, 56:41-61, 2011.

[FLF11] Bengt Fornberg, Elisabeth Larsson, and Natasha Flyer. Stable computations with gaussian radial basis functions. SIAM Journal on Scientific Computing, 33(2):869-892, 2011.

[GMP05] Assefaw H. Gebremedhin, Fredrik Manne, and Alex Pothen. What color is your jacobian? Graph coloring for computing derivatives. SIAM Review, 47(4):629-705, 2005.

[Gra69] Ronald L. Graham. Bounds on multiprocessing timing anomalies. SIAM Journal on Applied Mathematics, 17(2):416-429, March 1969.

[GWM14] Tony H Grubesic, Ran Wei, and Alan T Murray. Spatial clustering overview and comparison: Accuracy, sensitivity, and computational expense. Annals of the Association of American Geographers, 104(6):1134-1156, 2014.

[HDTC16] A. Hohl, E. Delmelle, W. Tang, and I. Casas. Accelerating the discovery of space-time patterns of infectious diseases using parallel computing. Spatial and Spatio-temporal Epidemiology, 2016.

[Hun13] Sascha Hunold. Scheduling moldable tasks with precedence constraints and arbitrary speedup functions on multiprocessors. In Prof of PPAM, pages 13-25, 2013.

[KCS04] M. P. Kwan, I. Casas, and B. Schmitz. Protection of geoprivacy and accuracy of spatial information: how effective are geographical masks? Cartographica: The International Journal for Geographic Information and Geovisualization, 39(2):15-28, 2004.

[KVKVO06] H. Karimabadi, H. X. Vu, D. Krauss-Varban, and Y. Omelchenko. Global hybrid simulations of the earth's magnetosphere. Numerical Modeling of Space Plasma Flows, December 2006.

[Lop15] Unai Lopez-Novoa. Contributions to the Efficient Use of General Purpose Coprocessors: Kernel Density Estimation as Case Study. PhD thesis, Universidad del País Vasco, 2015.

[LTW02] Renaud Lepère, Denis Trystram, and Gerhard J. Woeginger. Approximation algorithms for scheduling malleable tasks under precedence constraints. Int. J. Found. Comput. Sci., 13(4):613-627, 2002.

[MS96] Fredrik Manne and Tor Sørevik. Partitioning an array onto a mesh of processors. In Proc of PARA '96, pages 467-477, 1996.

[MXYB15] William B March, Bo Xiao, Chenhan D Yu, and George Biros. ASKIT: an efficient, parallel library for high-dimensional kernel summations. SIAM Journal on Scientific Computing (to appear), 2015.

[Nic94] David Nicol. Rectilinear partitioning of irregular data parallel computations. JPDC, 23:119-134, 1994.

[NY10] T. Nakaya and K. Yano. Visualising crime clusters in a space-time cube: an exploratory data-analysis approach using space-time kernel density estimation and scan statistics. Transactions in GIS, 14(3):223$239,2010$.

[PA97] Ali Pinar and Cevdet Aykanat. Sparse matrix decomposition with optimal load balancing. In Proc. of HiPC 1997, 1997.

[Sil86] B. W. Silverman. Density estimation for statistics and data analysis, volume 26. CRC press, 1986.

[USZS96] Manuel Ujaldon, Shamik Sharma, Emilio Zapata, and Joel Saltz. Experimental evaluation of efficient sparse matrix distributions. In Proc. of SuperComputing'96, 1996.

[YDGD03] Changjiang Yang, Ramani Duraiswami, Nail A Gumerov, and Larry Davis. Improved fast gauss transform and efficient kernel density estimation. In Proceedings of the Ninth IEEE International Conference on Computer Vision, pages 664-671. IEEE, 2003. 